\section{Sedimentação da avaliação de tecnologias em saúde em hospitais: uma revisão de escopo}

\author{
Sedimentation of health technology assessment \\ in hospitals: a scoping review
}

\section{Sedimentación en la evaluación de tecnologías de salud en hospitales: una revisión de alcance}

Johnathan Portela da Silva Galdino 1 Erika Barbosa Camargo 1

Flavia Tavares Silva Elias 1

doi: $10.1590 / 0102-311 \times 00352520$

\section{Resumo}

O objetivo do estudo foi analisar o nível de sedimentação da avaliação de tecnologias em saúde (ATS) hospitalar em diversos contextos. Foi realizada revisão de escopo segundo metodologia do Instituto Joanna Briggs, cujo modelo de análise dos dados foi composto pela combinação das dimensões de estrutura, processo e resultado de Donabedian e das dimensões do projeto Adopting Hospital Based Health Technology Assessment in European Union (AdHopHTA). Foram identificados 270 estudos, após remoção de duplicatas e leitura de textos completos, 36 referências atenderam aos critérios de elegibilidade. Trinta e seis hospitais foram identificados, sendo 24 hospitais de grande porte com capacidade extra de leitos. Vinte e três hospitais tinham vínculos universitários. Destaque ao Canadá, com cinco hospitais universitários, sendo quatro com financiamento público. Metade dos hospitais identificados tinham unidades de ATS hospitalar (18/36). Hospitais com nível sedimentado corresponderam a 75\% (27/36) e parcialmente sedimentado a 25\% (9/36). Não houve hospital com sedimentação incipiente. Mensurar o nível de sedimentação da ATS nos hospitais identificados contribui para o entendimento de como a inserção ocorre no campo da ATS hospitalar. Neste estudo, mostrou-se a importância de identificar fatores como sustentabilidade, crescimento e evolução da ATS hospitalar em países com e sem tradição com o tema.

Avaliação da Tecnologia Biomédica; Hospitais; Gestão em Saúde;

Tomada de Decisão; Governança

\section{Correspondência}

F. T. S. Elias

Gerência Regional de Brasília, Fundação Oswaldo Cruz. Av. L3 Norte s/n, Gleba A, 1o andar, Campus Darcy Ribeiro, Brasilia, DF 70904-130, Brasil.

flavia.elias@fiocruz.br

1 Gerência Regional de Brasília, Fundação Oswaldo Cruz, Brasília, Brasil. 


\section{Introdução}

A avaliação de tecnologias em saúde (ATS) tem criado diferentes processos de incorporação para cobertura de tecnologias nos serviços de saúde ${ }^{1}$. Cada um dos diversos países tem experimentado mecanismos de ATS de forma adaptada 2,3 aos seus contextos, tendo em vista a transição nutricional 4 , demográfica e epidemiológica 5 , as pressões econômicas e tecnológicas 6 , a expansão da oferta de tecnologias e os escassos investimentos financeiros 1,7,8.

A alta demanda das tecnologias em ambientes hospitalares 9 exigem informações para orientar o processo decisório na otimização do uso dos avanços tecnológicos 9 . Muitos fatores influenciam esse processo, como expectativas da sociedade por novas tecnologias e alta demanda por cuidados, aumento dos custos relacionados à falta de racionalidade técnica na aquisição de tecnologias e, consequentemente, impacto orçamentário e organizacional, a curto e a longo prazo, sobre as finanças hospitalares e para a assistência ao paciente 10 .

A utilização da ATS em hospitais contribui para tomada de decisão, capacitação profissional, aproximação do conhecimento técnico ao científico, economia de recursos e parcerias 11,12,13,14,15. Os hospitais são estratégicos para o campo da gestão clínica e qualidade do cuidado 10, aberto a parcerias com agências e grupos nacionais e internacionais 3 , como o subgrupo hospital-base de atenção de tecnologia em saúde (hospital-based health technology assessment - HB-HTA), criado em 2006 pela Sociedade Internacional de ATS 5 . Reconhecer o hospital nesse local estratégico fez com que as redes de agências de ATS mobilizassem conhecimento e ferramentas para melhorar a gestão hospitalar.

Traduzida livremente do termo internacional HB-HTA, o uso da ATS hospitalar é expressivo em todo o mundo ${ }^{1}$, pois permite desenvolver processos, lideranças, ferramentas e métodos de boas práticas em ATS adaptados ao contexto hospitalar 2,3 para melhoria de tratamentos, diagnósticos, serviços e otimização dos recursos hospitalares 2 .

Em que pese o movimento HB-HTA, a aplicação incipiente da ATS no processo de decisão hospitalar está ligada ao incipiente planejamento $7 \mathrm{e}$ mundialmente ainda existem fragilidades 1 . Destacamse a escassez de recursos e orçamento próprio para atividades de ATS, falta de equipe qualificada para gestão de evidências úteis aos processos decisórios, bloqueio à utilização das evidências e resistência à mudança por parte de gestores e profissionais 1,2,9,10,16. Para minimizar essas barreiras, a exploração de modelos organizacionais 2 pode ser uma estratégia de reorganização da HB-HTA.

A combinação das dimensões de estrutura, processo e resultado de Donabedian 17,18 e das dimensões do projeto Adopting Hospital Based Health Technology Assessment in European Union (AdHopHTA) 2,3 podem apoiar a definição de modelos propícios para o contexto de países como o Brasil, onde também são observadas barreiras. Nesse sentido, este estudo tem como objetivo analisar o nível de sedimentação da ATS hospitalar no contexto nacional e internacional.

\section{Método}

Trata-se de uma revisão de escopo para descobrir o nível de sedimentação da ATS hospitalar em experiências nacionais e internacionais. O modelo de análise foi composto pela combinação das dimensões de estrutura, processo e resultado de Donabedian 17,18 e das dimensões do projeto AdHopHTA 2,3.

Optou-se pela revisão de escopo para examinar a extensão e a natureza das publicações que reportavam as experiências de ATS em âmbito hospitalar, possibilitando analisar o nível de sedimentação em diferentes contextos.

Para fins deste estudo, entende-se como "sedimentação" a disposição de recursos, processos, métodos e ações para implementação efetiva da ATS no contexto hospitalar, considerando as caraterísticas que definem uma unidade de ATS hospitalar, ou seja, sedimentadas na formalização, especialização, integração, autoridade e profissionalização 2,19.

Esta revisão segue o checklist Preferred Reporting Items for Systematic reviews and Meta-Analyses extension for Scoping Reviews (PRISMA-ScR) 20,21. O protocolo foi registrado na Open Science Framework (https://osf.io/jtmsc). 


\section{Fontes de informação e estratégia de busca}

Foram utilizadas: Biblioteca Virtual em Saúde (BVS), MEDLINE/PubMed, Web of Science, SciVerse Scopus (Elsevier) e Embase, e as buscas com o termo (MeSH) "hospital" em consonância com o termo livre e "Hospital-Based Health Technology Assessment". A busca foi realizada em 23 de novembro de 2019 (Material suplementar: http://cadernos.ensp.fiocruz.br/static//arquivo/suppl-e-00352520port_9513.pdf). Publicações adicionais foram encontradas nas referências bibliográficas dos artigos incluídos.

\section{Critérios de elegibilidade e processos de seleção}

Foram considerados artigos completos sobre experiências relacionadas à gestão, governança, estrutura e organização da ATS em hospitais. A busca não foi limitada por país, ano de publicação ou idioma. Foram excluídos resumos e artigos que não abordavam contextos específicos de hospitais e com ênfase em resultados de eficácia e segurança de tecnologias específicas, salvo quando apresentavam experiências com critérios e modelos de avaliação utilizados pelos hospitais identificados. Utilizouse o software Mendeley (https://www.mendeley.com/?interaction_required=true) para remoção de duplicatas. A leitura de título e resumo foi realizada por dois revisores (J.P.S.G., F.T.S.E.) por meio do software Rayyan Qatar Computing Research Institute (Rayyan QCRI. https://www.rayyan.ai/) e o texto completo por um revisor (J.P.S.G.). Também houve outro para resolução de dúvidas (E.B.C.).

\section{Extração de dados}

A extração de dados foi realizada por um revisor (J.P.S.G.) e outras duas revisoras (F.T.S.E., E.B.C.) foram consultadas em caso de dúvidas sobre a classificação dos hospitais identificados. Utilizou-se o software Microsoft Excel (https://products.office.com/) para construção da tabela de extração. As variáveis analisadas foram: título, autor, ano, país, tipo de estudo, hospital identificado, porte e perfil assistencial do hospital, modelo organizacional de ATS hospitalar e outras dimensões de análise. O porte e perfil assistencial foi analisado em buscas nos sítios eletrônicos dos hospitais identificados. $\mathrm{O}$ porte do hospital 22 se refere à quantidade de leitos disponibilizada, sendo os com até 50 leitos considerados de pequeno porte; de 51 a 150 leitos de médio porte; de 151 a 500 leitos de grande porte; e com 500 leitos ou mais de grande porte com capacidade extra de leitos. Para o perfil assistencial dos estabelecimentos 22 , utilizou-se hospital universitário, especializado ou de ensino e pesquisa, conforme descrições nos sítios eletrônicos dos hospitais identificados.

A variável modelo organizacional de ATS hospitalar baseou-se na estrutura do The AdHopHTA Handbook 2,23, correspondendo às categorias: "modelo embaixador ou grupo autônomo", formadores de opinião que agem de maneira ainda individualizada e informal; "grupo matricial de ATS ou mini-ATS", profissionais com experiência em ATS que utilizam evidências relevantes (clínicos, econômicos, epidemiológicos e organizativos) para informar tomadores de decisão, podendo envolver colaboradores internos e externos; "comitê interno" multidisciplinar com revisão de evidências, perspectivas e recomendações internas padronizadas; "unidade de ATS hospitalar", estrutura formal dedicada à ATS com equipe própria em tempo integral, produção de material de alta qualidade sobre incorporação de tecnologias em saúde e interface externa com outras redes ou instituições.

Vinte e uma dimensões de análise foram identificadas e distribuídas nos eixos estrutura, processo e resultado. Foram considerados no eixo estrutura os aspectos financeiros, legais e normativos, sistemas para compartilhamento de informações, modelos vigentes, governança e parcerias para desenvolvimento da ATS hospitalar. No eixo processo, ficaram as ferramentas para pesquisa, desenvolvimento e monitoramento da ATS, e no eixo resultado os indicadores financeiros, capacitações, mudanças na organização clínica e gerencial e desafios para sustentabilidade da ATS nos serviços de saúde 17,18.

As 21 dimensões de análise foram classificadas em consonância com as dimensões para boas práticas em ATS hospitalar do projeto AdHopHTA 2,3: Processo avaliativo (D1); Liderança, estratégia e parcerias (D2); Recursos (D3); e Impacto (D4), conforme modelo teórico (Figura 1). 
Figura 1

Modelo para caracterização dos estudos incluídos no escopo da revisão de avaliação de tecnologias em saúde (ATS) hospitalar.

\begin{tabular}{|c|c|c|}
\hline EIXO & DIMENSÕES DE ANÁLISE & ESCORE \\
\hline \multirow{7}{*}{ 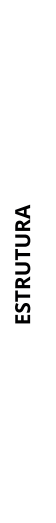 } & $\begin{array}{l}\text { ASPECTOS_ECONÔMICOS: Recursos financeiros do hospital identificado ou busca por fundos adicionais para cobrir os } \\
\left.\text { custos operacionais e garantir um local de trabalho adequado (D1 e D } 3^{\star}\right) .1 \text { ponto. }\end{array}$ & \multirow{7}{*}{$\begin{array}{l}\text { Máximo }=7 \text { pontos } \\
\text { - } 1 \text { ou } 2 \text { pontos }= \\
\text { incipiente }(0,0) \\
\text { - } 3,4 \text { ou } 5 \text { pontos }= \\
\text { parcial }(0,5) \\
\text { - } 6 \text { ou } 7 \text { pontos }= \\
\text { sedimentado }(1,0)\end{array}$} \\
\hline & ASPECTOS_LEGAIS_NORM: Normas existentes para realização das atividades de ATS nos hospitais (D2*). 1 ponto. & \\
\hline & $\begin{array}{l}\text { SISTEMAS_INFO: Sistemas de informação para compartilhamento de dados relevantes e métodos da ATS aos gestores e } \\
\text { partes interessadas dos hospitais (D1*). } 1 \text { ponto. }\end{array}$ & \\
\hline & $\begin{array}{l}\text { HOSP_ÁREAS_ATS: Matriciamento, relações dos grupos com as demais áreas do hospital. Modelos de ATS realizados: } \\
\text { modelo embaixador, grupo matricial ou mini-ATS, comitê interno ou unidade de ATS hospitalar (estruturada) (D2*). } 1 \text { ponto. }\end{array}$ & \\
\hline & $\begin{array}{l}\text { GOVERNANÇA_ATS: Formalização da ATS no organograma do hospital identificado. A missão, a visão e os valores dos grupos ou } \\
\text { unidades ATS estão definidos e são coerentes com a missão e estratégia geral do hospital, o que permite uma governança } \\
\text { clara da atividade ATS hospitalar (D2*). } 1 \text { ponto. }\end{array}$ & \\
\hline & $\begin{array}{l}\text { PARCERIAS_REDE: Parcerias e colaborações, internas ou externas, para compartilhamento de recursos e realização da ATS } \\
\text { hospitalar (D2*). } 1 \text { ponto. }\end{array}$ & \\
\hline & $\begin{array}{l}\text { PERFIS_DEFINIDOS: Definição de perfis, habilidades profissionais, política de recrutamento e planos de desenvolvimento de } \\
\text { carreira no campo da ATS hospitalar (D3*). } 1 \text { ponto. }\end{array}$ & \\
\hline \multirow{7}{*}{ 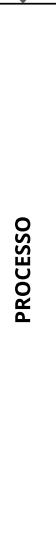 } & $\begin{array}{l}\text { ESCOPO_CONTEXTO: Definição do escopo para formulação da pergunta, com base no contexto local estudado por stakeholders. } \\
\text { Objetivo da pesquisa e definição de público-alvo ou priorização para elaboração de estudos no campo da ATS hospitalar } \\
\text { (D1*). } 1 \text { ponto. }\end{array}$ & \multirow{7}{*}{$\begin{array}{l}\text { Máximo }=7 \text { pontos } \\
\text { - } 1 \text { ou } 2 \text { pontos }= \\
\text { incipiente }(0,0) \\
\text { - } 3,4 \text { ou } 5 \text { pontos }= \\
\text { parcial }(0,5) \\
\text { - } 6 \text { ou } 7 \text { pontos }= \\
\text { sedimentado }(1,0)\end{array}$} \\
\hline & $\begin{array}{l}\text { LIDERANÇA_ATS: Diretrizes para liderança, organização do serviço e formulação de estratégias de comunicação em atividades } \\
\text { internas e externas de ATS (D2*). } 1 \text { ponto. }\end{array}$ & \\
\hline & $\begin{array}{l}\text { PESQUISA_DESENV: Pesquisa, desenvolvimento e monitoramento de tecnologias em saúde. Critérios para seleção e/ou } \\
\text { priorização de tecnologias em saúde a serem avaliadas, baseados no contexto definido (D4*). } 1 \text { ponto. }\end{array}$ & \\
\hline & $\begin{array}{l}\text { PARTICIPAÇÃO_SOCIAL: Registro e/ou uso de preferências de pacientes por tecnologias em saúde, participação social na } \\
\text { avaliação e elaboração de recomendações em ATS (D1*). } 1 \text { ponto. }\end{array}$ & \\
\hline & $\begin{array}{l}\text { RELATÓRIO_ATS: Relatórios de ATS e/ou demais documentos para recomendação de tecnologias em saúde utilizadas no } \\
\text { hospital identificado (D1*). } 1 \text { ponto. }\end{array}$ & \\
\hline & $\begin{array}{l}\text { MÉTODO_REPLICÁVEL: Metodologia prevê qualidade ou condição transferivel e adequabilidade dos estudos para outros } \\
\text { hospitais (D1*). } 1 \text { ponto. }\end{array}$ & \\
\hline & $\begin{array}{l}\text { INFO_TRAD_GESTORES: Gestão da informação ou tradução do conhecimento sobre as possibilidades de utilização das } \\
\text { ferramentas de ATS na decisão de gestores e stakeholders (D1*). } 1 \text { ponto. }\end{array}$ & \\
\hline \multirow{7}{*}{ 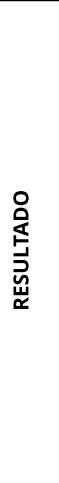 } & RESULT_FINANCEIRO: Indicadores e/ou resultados da ATS sobre os recursos financeiros no hospital identificado (D4*). 1 ponto. & \multirow{7}{*}{$\begin{array}{l}\text { Máximo }=7 \text { pontos } \\
\text { - } 1 \text { ou } 2 \text { pontos }= \\
\text { incipiente }(0,0) \\
\text { - } 3,4 \text { ou } 5 \text { pontos }= \\
\text { parcial }(0,5) \\
\text { - } 6 \text { ou } 7 \text { pontos }= \\
\text { sedimentado }(1,0)\end{array}$} \\
\hline & $\begin{array}{l}\text { RESULT_TREIN_PROF: Indicadores e/ou resultados da ATS sobre recursos humanos, treinamentos e profissionais capacitados no } \\
\text { hospital identificado (D3*). } 1 \text { ponto. }\end{array}$ & \\
\hline & $\begin{array}{l}\text { RESULT_SERVIÇO: Indicadores e/ou resultados da ATS na organização (gestora ou clínica) dos serviços de saúde do hospital } \\
\text { identificado (D4*). } 1 \text { ponto. }\end{array}$ & \\
\hline & $\begin{array}{l}\text { RESULT_RECOMEND: Indicadores e/ou resultados da ATS na produção de informações sobre avaliações e recomendaç̃̃es de } \\
\text { tecnologias em saúde no hospital identificado (D4*). } 1 \text { ponto. }\end{array}$ & \\
\hline & $\begin{array}{l}\text { RESULT_SATISFAÇÃO: Satisfação de clínicos, gestores ou partes interessadas ante a implementação das ferramentas de ATS no } \\
\text { hospital identificado (D4*). } 1 \text { ponto. }\end{array}$ & \\
\hline & BARREIRAS_IMPLEM: Barreiras e limitações para implementação da ATS hospitalar identificadas (D1*). 1 ponto. & \\
\hline & SUSTENT_CREDIB: Sustentabilidade dos recursos de ATS no hospital identificado e reconhecimento interno e externo (D4*). 1 ponto. & \\
\hline & ão da ATS no contexto hospitalar & \\
\hline
\end{tabular}

* Dimensões do modelo Adopting Hospital Based Health Technology Assessment in European Union (AdHopHTA) 2,3.

Fonte: elaboração própria. 


\section{Análise dos dados}

Os hospitais identificados nas referências selecionadas receberam um ponto para cada uma das vinte e uma dimensões de análise (Figura 1), caso apresentassem a informação correspondente. Nos casos que apresentaram mais de um hospital identificado, o ponto da dimensão de análise foi dividido pelo número de hospitais identificados. Um grupo focal de três especialistas na área de ATS por meio de consenso harmonizou os 21 pontos das dimensões de análise entre os eixos, estrutura, processo e resultado, resultando em até sete pontos para cada eixo. Se o eixo resultou em 1 ou 2, foi caracterizado como incipiente (pontuação 0,0); de 3 a 5 como parcial (pontuação 0,5); e 6 ou 7 como sedimentado (pontuação 1,0). Após análise e somatório dos dados, foi verificada a sedimentação das ações de ATS nos hospitais identificados em três níveis 2 : "sedimentação incipiente" ( 0,0 ou 0,5 pontos finais), que corresponde à incipiente ou baixa sustentação da proposta da ATS nos hospitais identificados; "parcialmente sedimentado" (1,0 ou 1,5 pontos finais), que não apresenta estrutura ou processo de ATS hospitalar totalmente amadurecido, mas apresenta resultados; e "sedimentado" (2,0, 2,5 ou 3,0 pontos finais), com estrutura, processo e resultados definitivos e formalizados (Figura 1).

\section{Resultados}

\section{Busca e seleção dos estudos}

No total, a busca identificou 270 estudos, restando 74 para leitura completa, sendo elegíveis 14 . Uma busca manual nas referências dos artigos elegíveis foi realizada e 22 estudos foram incluídos na pesquisa, totalizando 36 referências (Figura 2).

\section{Características dos estudos incluídos}

Os 36 estudos incluídos foram publicados entre 2005 e 2019, com 58,3\% publicados em 2016. A referência especializada para extração dos estudos adicionais foi o livro Hospital-Based Health Technology Assessment: the Next Frontier for Health Technology Assessment 1, o que resultou no maior quantitativo de estudos publicados no ano de 2016. Dezenove países foram identificados. Artigos e capítulos de livro compuseram os estudos incluídos na pesquisa e cinco estudos $24,25,26,27,28$ contextualizaram mais de um hospital por estudo.

Foram identificados 36 hospitais nos estudos incluídos (Quadro 1). Cinco hospitais canadenses Centro Hospitalar da Universidade de Sherbrooke (Centre Hospitalier Universitaire de Sherbrooke - H11), Centro de Saúde da Universidade McGill (McGill University Health Centre - H12), Centro Hospitalar da Universidade de Montreal (Centre Hospitalier de l'Université de Montréal - H13), Hospital Universitário de Quebec-Laval University (Centre Hospitalier Universitaire de Québec-Université Laval - H14) e Hospital para Crianças Peter Gilgan, Centro de Investigação e Aprendizagem (Hospital for Sick Children Peter Gilgan Centre for Research and Learning - H15) - foram maioria dentre os identificados, totalizando 13,9\% (5/36). O Brasil apresentou três hospitais (3/36): Hospital das Clínicas da Faculdade de Medicina de Ribeirão Preto da Universidade de São Paulo (H8), Instituto Nacional de Cardiologia (H9) e Hospital Nossa Senhora da Conceição S.A. (H10).

Quanto ao porte hospitalar, hospitais de grande porte com capacidade extra de leitos (HGPE) foram maioria e corresponderam a 66,7\% (24/36), seguidos de hospitais de grande porte (HGP), com $25 \%$ (9/36), e hospitais de médio porte (HMP), com 8,3\% (3/36). Em relação ao Brasil, dois eram HGP e um HGPE (3/36). Não foram identificados hospitais de pequeno porte (HPP).

Quanto ao perfil assistencial, 23 hospitais tinham vínculos universitários, com destaque para HGPE, com 82,6\% (19/23). Nove hospitais eram especializados e a maioria era HGP com 44,4\% (4/9). Quatro hospitais de ensino e pesquisa que não possuíam ou não especificaram vínculo com universidades foram identificados, sendo a maioria HGPE, com 75\% (3/4). Do total de hospitais identificados, 19 eram HGPE com vínculos universitários e 89,5\% (17/19) tinha financiamento público. Destaque ao Canadá, com quatro hospitais universitários de financiamento público. No Brasil, os hospitais eram universitários e de ensino e pesquisa com financiamento público (3/36). 
Figura 2

Fluxograma PRISMA-ScR do processo de seleção dos estudos.

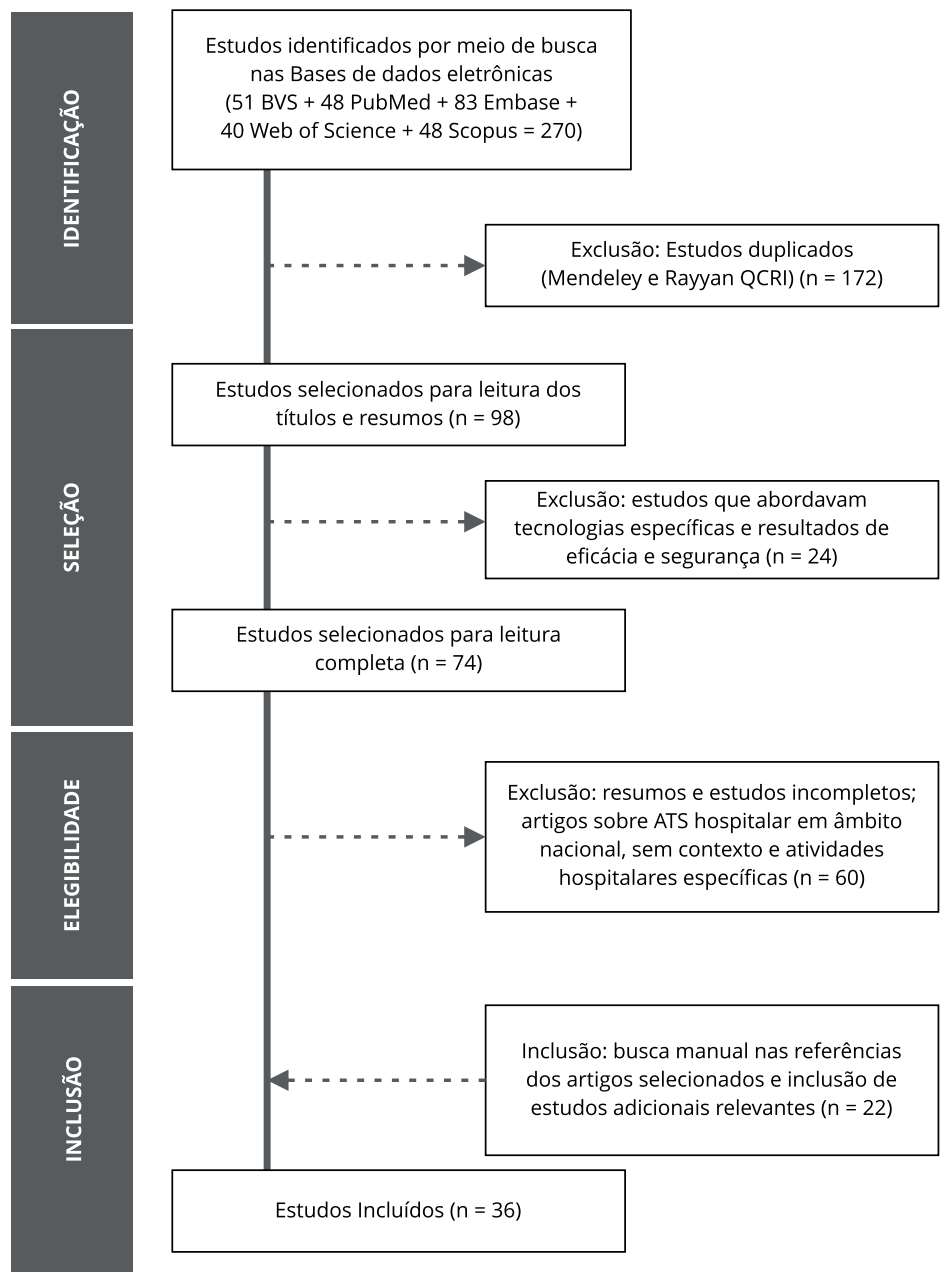

ATS: avaliação de tecnologia em saúde; BVS: Biblioteca Virtual em Saúde; PRISMA-ScR: Preferred Reporting Items for Systematic Reviews and Meta-Analyses extension for Scoping Reviews.

Fonte: elaboração própria, conforme checklist PRISMA-SCR 20,21.

Quanto ao modelo organizacional de ATS, metade dos hospitais identificados tinham unidades de ATS hospitalar, com 50\% (18/36), seguidos por grupo matricial de ATS ou mini-ATS, com 19,4\% (7/36); unidade de ATS hospitalar combinada ao modelo comitê interno, com 16,7\% (6/36); comitê interno, com 11,1\% (4/36); e modelo embaixador ou grupo autônomo, com 2,8\% (1/36). Dois hospitais brasileiros possuíam unidades de ATS hospitalar e um com o modelo grupo matricial de ATS ou mini-ATS (3/36).

\section{Nível de sedimentação das ações de avaliação de tecnologias em saúde nos hospitais identificados}

Constatou-se, nos estudos analisados (Quadro 1), que 75\% (27/36) dos hospitais identificados foram caracterizados como hospitais com nível sedimentado e 25\% (9/36) com nível parcialmente sedimentado segundo os critérios adotados. O estudo não identificou hospitais com o nível de sedimentação incipiente (Tabela 1). 


\section{Quadro 1}

Características dos estudos incluídos e hospitais identificados.

\begin{tabular}{|c|c|c|c|c|c|}
\hline PAís & $\begin{array}{l}\text { ESTUDO/ } \\
\text { ANO }\end{array}$ & AUTOR & HOSPITAL IDENTIFICADO & $\begin{array}{l}\text { PORTE E PERFIL } \\
\text { ASSISTENCIAL }\end{array}$ & $\begin{array}{c}\text { MODELO } \\
\text { ORGANIZACIONAL DE } \\
\text { ATS HOSPITALAR }\end{array}$ \\
\hline $\begin{array}{l}\text { África do } \\
\text { Sul }\end{array}$ & E1/2016 & $\begin{array}{c}\text { Mueller \& } \\
\text { Govender } 48\end{array}$ & $\begin{array}{l}\text { Hospital Acadêmico Charlotte } \\
\text { Maxeke de Joanesburgo (H1) }\end{array}$ & $\begin{array}{l}\text { HGPE público } \\
\text { universitário }\end{array}$ & Unidade de ATS hospitalar \\
\hline \multirow[t]{3}{*}{ Argentina } & $E 2 / 2015$ & Demirdjian 9 & Hospital de Pediatria Garrahan ( $\mathrm{H} 2)$ & $\begin{array}{l}\text { HGPE público } \\
\text { especializado }\end{array}$ & Unidade de ATS hospitalar \\
\hline & \multirow[t]{2}{*}{ E3/2016 } & \multirow[t]{2}{*}{ Demirdjian et al. 24} & Hospital de Pediatria Garrahan (H2) & \multirow{2}{*}{$\begin{array}{l}\text { HMP público } \\
\text { especializado }\end{array}$} & \multirow[t]{2}{*}{ Comitê interno } \\
\hline & & & Hospital El Cruce (H3) & & \\
\hline \multirow[t]{4}{*}{ Austrália } & \multirow[t]{4}{*}{$E 4 / 2011$} & \multirow[t]{4}{*}{ Saaid et al. 25} & Hospital A (H4) & $\begin{array}{l}\text { HGP privado sem } \\
\text { fins lucrativos } \\
\text { especializado }\end{array}$ & $\begin{array}{c}\text { Grupo matricial de ATS ou } \\
\text { mini-ATS }\end{array}$ \\
\hline & & & Hospital B (H5) & $\begin{array}{l}\text { HMP privado sem } \\
\text { fins lucrativos } \\
\text { especializado }\end{array}$ & $\begin{array}{c}\text { Grupo matricial de ATS ou } \\
\text { mini-ATS }\end{array}$ \\
\hline & & & Hospital C (H6) & $\begin{array}{l}\text { HMP privado sem } \\
\text { fins lucrativos } \\
\text { especializado }\end{array}$ & $\begin{array}{c}\text { Grupo matricial de ATS ou } \\
\text { mini-ATS }\end{array}$ \\
\hline & & & Hospital D (H7) & $\begin{array}{l}\text { HGP público } \\
\text { especializado }\end{array}$ & $\begin{array}{c}\text { Grupo matricial de ATS ou } \\
\text { mini-ATS }\end{array}$ \\
\hline \multirow[t]{3}{*}{ Brasil } & \multirow[t]{3}{*}{$E 5 / 2013$} & Nunes et al. 5 & $\begin{array}{l}\text { Hospital das Clínicas, Faculdade } \\
\text { de Medicina de Ribeirão Preto, } \\
\text { Universidade de São Paulo (H8) }\end{array}$ & $\begin{array}{l}\text { HGP público } \\
\text { universitário }\end{array}$ & $\begin{array}{c}\text { Grupo matricial de ATS ou } \\
\text { mini-ATS }\end{array}$ \\
\hline & & \multirow[t]{2}{*}{ Santos et al. 26} & $\begin{array}{l}\text { Instituto Nacional de Cardiologia } \\
\qquad(\mathrm{H} 9)\end{array}$ & $\begin{array}{l}\text { HGP público de } \\
\text { ensino e pesquisa }\end{array}$ & Unidade de ATS hospitalar \\
\hline & & & $\begin{array}{c}\text { Hospital Nossa Senhora da } \\
\text { Conceição S.A. (Grupo Hospitalar } \\
\text { Conceição) }(\mathrm{H} 10)\end{array}$ & $\begin{array}{l}\text { HGPE público de } \\
\text { ensino e pesquisa }\end{array}$ & Unidade de ATS hospitalar \\
\hline \multirow[t]{10}{*}{ Canadá } & $E 7 / 2016$ & Bellemare et al. 32 & \multirow{4}{*}{$\begin{array}{c}\text { Centro Hospitalar da Universidade } \\
\text { de Sherbrooke }(\mathrm{H} 11)\end{array}$} & \multirow{4}{*}{$\begin{array}{l}\text { HGPE público } \\
\text { universitário e } \\
\text { especializado }\end{array}$} & \multirow[t]{4}{*}{ Unidade de ATS hospitalar } \\
\hline & E8/2017 & Poder 33 & & & \\
\hline & $\mathrm{E} 9 / 2018$ & Poder et al. 34 & & & \\
\hline & $\mathrm{E} 10 / 2019$ & Poder et al. 35 & & & \\
\hline & $\mathrm{E} 11 / 2005$ & $\begin{array}{c}\text { McGregor \& } \\
\text { Brophy } 11\end{array}$ & \multirow[t]{3}{*}{$\begin{array}{l}\text { Centro de Saúde da Universidade } \\
\qquad \text { McGill }(\mathrm{H} 12)\end{array}$} & \multirow{3}{*}{$\begin{array}{l}\text { HGPE público } \\
\text { universitário e } \\
\text { especializado }\end{array}$} & \multirow[t]{3}{*}{ Unidade de ATS hospitalar } \\
\hline & $\mathrm{E} 12 / 2005$ & McGregor 12 & & & \\
\hline & $\mathrm{E} 13 / 2019$ & Almeida et al. 13 & & & \\
\hline & $\mathrm{E} 14 / 2016$ & Lepanto 31 & $\begin{array}{c}\text { Centro Hospitalar da Universidade } \\
\text { de Montreal (H13) }\end{array}$ & $\begin{array}{l}\text { HGPE público } \\
\text { universitário e } \\
\text { especializado }\end{array}$ & $\begin{array}{c}\text { Unidade de ATS hospitalar } \\
\text { e comitê interno }\end{array}$ \\
\hline & $\mathrm{E} 15 / 2016$ & Rhainds et al. 36 & $\begin{array}{l}\text { Hospital Universitário de Quebec- } \\
\text { Universidade de Laval }(\mathrm{H} 14)\end{array}$ & $\begin{array}{l}\text { HGPE público } \\
\text { universitário e } \\
\text { especializado }\end{array}$ & Unidade de ATS hospitalar \\
\hline & $E 16 / 2016$ & Ungar 37 & $\begin{array}{c}\text { Hospital para Crianças Peter } \\
\text { Gilgan, Centro de Investigação e } \\
\text { Aprendizagem (H15) }\end{array}$ & $\begin{array}{l}\text { HGP público } \\
\text { universitário e } \\
\text { especializado }\end{array}$ & Unidade de ATS hospitalar \\
\hline
\end{tabular}

(continua) 
Quadro 1 (continuação)

\begin{tabular}{|c|c|c|c|c|c|}
\hline PAís & $\begin{array}{l}\text { ESTUDO/ } \\
\text { ANO }\end{array}$ & AUTOR & HOSPITAL IDENTIFICADO & $\begin{array}{l}\text { PORTE E PERFIL } \\
\text { ASSISTENCIAL }\end{array}$ & $\begin{array}{l}\text { MODELO } \\
\text { ORGANIZACIONAL DE } \\
\text { ATS HOSPITALAR }\end{array}$ \\
\hline \multirow[t]{2}{*}{ Cazaquistão } & E17/2016 & $\begin{array}{c}\text { Kosherbayeva } \\
\text { et al. } 16\end{array}$ & $\begin{array}{l}\text { First General City Hospital of Astana } \\
\qquad(\mathrm{H} 16)\end{array}$ & $\begin{array}{l}\text { HGPE privado } \\
\text { especializado }\end{array}$ & Unidade de ATS hospitalar \\
\hline & $\mathrm{E} 18 / 2018$ & Avdeyev et al. 38 & $\begin{array}{l}\text { Medical Centre Hospital of the } \\
\text { President's Affairs Administration of } \\
\text { the Republic of Kazakhstan ( } \mathrm{H} 17)\end{array}$ & $\begin{array}{l}\text { HGP privado } \\
\text { especializado }\end{array}$ & Unidade de ATS hospitalar \\
\hline Dinamarca & E19/2016 & Kidholm \& Ølholm 29 & Hospital Universitário Odense (H18) & $\begin{array}{l}\text { HGPE público } \\
\text { universitário e } \\
\text { especializado }\end{array}$ & Unidade de ATS Hospitalar \\
\hline \multirow[t]{3}{*}{ Espanha } & \multirow[t]{3}{*}{ E20/2016 } & \multirow[t]{3}{*}{$\begin{array}{l}\text { Sampietro-Colom } \\
\text { et al. } 27\end{array}$} & $\begin{array}{c}\text { Hospital Clínico e Provincial de } \\
\text { Barcelona (H19) }\end{array}$ & $\begin{array}{l}\text { HGPE público } \\
\text { universitário e } \\
\text { especializado }\end{array}$ & Unidade de ATS hospitalar \\
\hline & & & $\begin{array}{l}\text { Hospital Universitario Virgen del } \\
\text { Rocío }(\mathrm{H} 20)\end{array}$ & $\begin{array}{l}\text { HGPE público } \\
\text { universitário e } \\
\text { especializado }\end{array}$ & $\begin{array}{c}\text { Unidade de ATS hospitalar } \\
\text { e comitê interno }\end{array}$ \\
\hline & & & $\begin{array}{l}\text { Hospital Sant Joan de Déu Barcelona } \\
\qquad(\mathrm{H} 21)\end{array}$ & $\begin{array}{l}\text { HGP público } \\
\text { universitário e } \\
\text { especializado }\end{array}$ & Comitê interno \\
\hline $\begin{array}{l}\text { Estados } \\
\text { Unidos }\end{array}$ & $E 21 / 2010$ & Mitchell et al. 39 & $\begin{array}{l}\text { Centro Médico Penn Presbyterian } \\
\qquad(\mathrm{H} 22)\end{array}$ & $\begin{array}{l}\text { HGP privado sem } \\
\text { fins lucrativos } \\
\text { especializado }\end{array}$ & $\begin{array}{c}\text { Unidade de ATS hospitalar } \\
\text { e comitê interno }\end{array}$ \\
\hline Finlândia & $E 22 / 2016$ & Pasternack \& Iris 47 & $\begin{array}{l}\text { Hospital Central da Universidade de } \\
\text { Helsinki }(\mathrm{H} 23)\end{array}$ & $\begin{array}{l}\text { HGPE privado sem } \\
\text { fins lucrativos } \\
\text { universitário } \\
\text { especializado }\end{array}$ & $\begin{array}{c}\text { Grupo matricial de ATS ou } \\
\text { mini-ATS }\end{array}$ \\
\hline França & $E 23 / 2016$ & Barna et al. 40 & Hospital Universitário de Paris (H24) & $\begin{array}{l}\text { HGPE público } \\
\text { universitário e } \\
\text { especializado }\end{array}$ & Unidade de ATS hospitalar \\
\hline $\begin{array}{l}\text { Países } \\
\text { Baixos }\end{array}$ & E24/2016 & van der Wilt et al. 49 & $\begin{array}{l}\text { Centro Médico Universidade } \\
\text { Radboud }(\mathrm{H} 25)\end{array}$ & $\begin{array}{l}\text { HGPE público } \\
\text { universitário }\end{array}$ & Unidade de ATS hospitalar \\
\hline Israel & $E 25 / 2019$ & Tal et al. 41 & $\begin{array}{c}\text { Centro Médico Yitzhak Shamir - } \\
\text { Assaf Harofeh (H26) }\end{array}$ & $\begin{array}{l}\text { HGPE público } \\
\text { universitário e } \\
\text { especializado }\end{array}$ & $\begin{array}{c}\text { Unidade de ATS hospitalar } \\
\text { e comitê interno }\end{array}$ \\
\hline \multirow[t]{4}{*}{ Itália } & $E 26 / 2005$ & Catananti et al. 14 & \multirow{2}{*}{$\begin{array}{c}\text { Hospital Universitário Agostino } \\
\text { Gemelli ( } \mathrm{H} 27)\end{array}$} & \multirow{2}{*}{$\begin{array}{l}\text { HGPE público } \\
\text { universitário e } \\
\text { especializado }\end{array}$} & \multirow[t]{2}{*}{ Unidade de ATS hospitalar } \\
\hline & $E 27 / 2016$ & $\begin{array}{l}\text { Marchetti \& } \\
\text { Cicchetti } 15\end{array}$ & & & \\
\hline & $E 28 / 2016$ & Miniati et al. 42 & $\begin{array}{l}\text { Hospital Universitário de Florença } \\
\text { - Hospital Universitário Careggi } \\
\text { AOUC }(\mathrm{H} 28)\end{array}$ & $\begin{array}{l}\text { HGPE público } \\
\text { universitário e } \\
\text { especializado }\end{array}$ & Unidade de ATS hospitalar \\
\hline & $E 29 / 2015$ & Manzi et al. 50 & Hospital Universitário Siena (H29) & $\begin{array}{l}\text { HGPE público } \\
\text { universitário e } \\
\text { especializado }\end{array}$ & $\begin{array}{c}\text { Unidade de ATS hospitalar } \\
\text { e comitê interno }\end{array}$ \\
\hline \multirow{2}{*}{$\begin{array}{l}\text { Nova } \\
\text { Zelândia }\end{array}$} & E30/2014 & Munn 43 & \multirow{2}{*}{$\begin{array}{c}\text { Conselho Distrital de Saúde de } \\
\text { Auckland - Hospital Municipal de } \\
\text { Auckland (H30) }\end{array}$} & \multirow{2}{*}{$\begin{array}{l}\text { HGPE público de } \\
\text { ensino e pesquisa e } \\
\text { especializado }\end{array}$} & \multirow{2}{*}{$\begin{array}{l}\text { Unidade de ATS hospitalar } \\
\text { e comitê interno }\end{array}$} \\
\hline & E31/2016 & Fitzgerald et al. 44 & & & \\
\hline
\end{tabular}

(continua) 


\begin{tabular}{|c|c|c|c|c|c|}
\hline PAís & $\begin{array}{l}\text { ESTUDO/ } \\
\text { ANO }\end{array}$ & AUTOR & HOSPITAL IDENTIFICADO & $\begin{array}{l}\text { PORTE E PERFIL } \\
\text { ASSISTENCIAL }\end{array}$ & $\begin{array}{c}\text { MODELO } \\
\text { ORGANIZACIONAL DE } \\
\text { ATS HOSPITALAR }\end{array}$ \\
\hline Singapura & E32/2016 & Pwee \& Chow 51 & Hospital Geral Changi (H31) & $\begin{array}{l}\text { HGPE público } \\
\text { universitário e } \\
\text { especializado }\end{array}$ & Comitê interno \\
\hline Suécia & E33/2016 & Jivegård et al. 45 & $\begin{array}{l}\text { Hospital Universitário Sahlgrenska } \\
\qquad(\mathrm{H} 32)\end{array}$ & $\begin{array}{l}\text { HGPE público } \\
\text { universitário e } \\
\text { especializado }\end{array}$ & $\begin{array}{c}\text { Grupo matricial de ATS ou } \\
\text { mini-ATS }\end{array}$ \\
\hline \multirow[t]{4}{*}{ Suíça } & E34/2016 & Grenon et al. 46 & $\begin{array}{l}\text { Hospital Universitário Lausanne } \\
\qquad(\mathrm{H} 33)\end{array}$ & $\begin{array}{l}\text { HGPE público } \\
\text { universitário e }\end{array}$ & Unidade de ATS hospitalar \\
\hline & \multirow[t]{3}{*}{$E 35 / 2016$} & \multirow[t]{3}{*}{$\begin{array}{l}\text { Wasserfallen \& } \\
\text { Pinget } 28\end{array}$} & $\begin{array}{l}\text { Hospital Universitário Lausanne } \\
\qquad(\mathrm{H} 33)\end{array}$ & especializado & \\
\hline & & & $\begin{array}{l}\text { Hospital Universitário Genebra } \\
\qquad(\mathrm{H} 34) ;\end{array}$ & $\begin{array}{l}\text { HGPE público } \\
\text { universitário e } \\
\text { especializado }\end{array}$ & Comitê interno \\
\hline & & & $\begin{array}{l}\text { Estabelecimentos do Hospital } \\
\text { Northern Vaudois (H35) }\end{array}$ & $\begin{array}{l}\text { HGP privado } \\
\text { especializado }\end{array}$ & $\begin{array}{l}\text { Modelo embaixador ou } \\
\text { grupo autônomo }\end{array}$ \\
\hline Turquia & E36/2016 & Kahveci et al. 30 & $\begin{array}{l}\text { Hospital de Formação e Investigação } \\
\text { Ankara Numune }(\mathrm{H} 36)\end{array}$ & $\begin{array}{c}\text { HGPE público de } \\
\text { ensino e pesquisa e } \\
\text { especializado }\end{array}$ & Unidade de ATS hospitalar \\
\hline
\end{tabular}

ATS: avaliação de tecnologias em saúde; HGP: hospital de grande porte; HGPE: hospital de grande porte com capacidade extra de leitos; HMP: hospital de médio porte.

Nota: E1 a E36: estudos incluídos na revisão. H1 a H36: hospitais identificados.

Fonte: elaboração própria.

\section{Hospitais sedimentados}

Dentre os 27 hospitais que apresentaram o nível sedimentado (Tabela 1), nove somaram maior pontuação na análise final (3,0 pontos) e apresentaram unidades de ATS hospitalar (H2 9,24, H18 29, H19 27, H27 14,15 e H36 30), comitês internos (H3 24 e H21 27) ou ambos os modelos (H13 31 e H20 27), apoiados pela administração para desenvolver relatórios e emitir recomendações acessíveis e traduzidas aos tomadores de decisão. Somente H36 não tem apoio totalmente formalizado para capacitação profissional e produção de trabalhos multidisciplinares 30. Todos os métodos utilizados para produção de relatórios e recomendações, adaptados aos contextos dos hospitais identificados, foram caracterizados como replicável a outros hospitais e com a implementação da ATS houve redução de custos e mudanças no processo de incorporação de tecnologias, com reconhecimento e suporte de profissionais e gestores 9,14,15,24,27,29,30,31. Tais hospitais atenderam a pelo menos seis dimensões de análise em cada um dos eixos estrutura, processo e resultado (Tabela 1).

Os hospitais com nível sedimentado de ATS tiveram representação de 14 países, sendo eles Argentina 9,24, Brasil 5,26, Canadá 11,12,13,30,32,33,34,35,36,37, Cazaquistão 16,38, Dinamarca 29, Espanha 27, Estados Unidos 39, França 40, Israel 41, Itália 14,15,42, Nova Zelândia 43,44, Suécia 45, Suíça 28,46 e Turquia 30.

O Canadá apresentou o maior número de estudos e de hospitais identificados. Dez estudos do Canadá destacaram quatro HGPE (H11 32,33,34,35, H12 11,12,13, H13 31 e H14 36) e um HGP (H15 37) universitários com unidades de ATS hospitalar. Todos os hospitais canadenses identificados apresentaram o nível sedimentado. 
Tabela 1

Nível de sedimentação dos hospitais identificados nos estudos incluídos.

\begin{tabular}{|c|c|c|c|c|}
\hline Hospitais & Estrutura & Processo & Resultado & $\begin{array}{c}\text { Nível de } \\
\text { sedimentação }\end{array}$ \\
\hline $\mathrm{H} 1$ & 0,5 & 0,5 & 0,5 & 1,5 \\
\hline $\mathrm{H} 2$ & 1,0 & 1,0 & 1,0 & 3,0 \\
\hline H3 & 1,0 & 1,0 & 1,0 & 3,0 \\
\hline $\mathrm{H} 4$ & 0,0 & 0,5 & 0,5 & 1,0 \\
\hline H5 & 0,0 & 0,5 & 0,5 & 1,0 \\
\hline H6 & 0,0 & 0,5 & 0,5 & 1,0 \\
\hline $\mathrm{H} 7$ & 0,0 & 0,5 & 0,5 & 2,0 \\
\hline $\mathrm{H} 8$ & 0,5 & 1,0 & 0,5 & 2,0 \\
\hline H9 & 1,0 & 0,5 & 1,0 & 2,5 \\
\hline $\mathrm{H} 10$ & 1,0 & 0,5 & 1,0 & 2,5 \\
\hline $\mathrm{H} 11$ & 1,0 & 0,5 & 0,5 & 2,0 \\
\hline $\mathrm{H} 12$ & 0,5 & 1,0 & 1,0 & 2,5 \\
\hline $\mathrm{H} 13$ & 1,0 & 1,0 & 1,0 & 3,0 \\
\hline $\mathrm{H} 14$ & 1,0 & 0,5 & 1,0 & 2,5 \\
\hline $\mathrm{H} 15$ & 0,5 & 1,0 & 1,0 & 2,5 \\
\hline H16 & 1,0 & 0,5 & 1,0 & 2,5 \\
\hline $\mathrm{H} 17$ & 1,0 & 1,0 & 0,5 & 2,5 \\
\hline $\mathrm{H} 18$ & 1,0 & 1,0 & 1,0 & 3,0 \\
\hline H19 & 1,0 & 1,0 & 1,0 & 3,0 \\
\hline $\mathrm{H} 2 \mathrm{O}$ & 1,0 & 1,0 & 1,0 & 3,0 \\
\hline $\mathrm{H} 21$ & 1,0 & 1,0 & 1,0 & 3,0 \\
\hline $\mathrm{H} 22$ & 0,5 & 0,5 & 1,0 & 2,0 \\
\hline $\mathrm{H} 23$ & 0,5 & 0,5 & 0,5 & 1,5 \\
\hline $\mathrm{H} 24$ & 1,0 & 0,5 & 0,5 & 2,0 \\
\hline $\mathrm{H} 25$ & 0,5 & 0,5 & 0,5 & 1,5 \\
\hline $\mathrm{H} 26$ & 1,0 & 0,5 & 0,5 & 2,0 \\
\hline $\mathrm{H} 27$ & 1,0 & 1,0 & 1,0 & 3,0 \\
\hline $\mathrm{H} 28$ & 0,5 & 1,0 & 1,0 & 2,5 \\
\hline $\mathrm{H} 29$ & 0,5 & 0,5 & 0,5 & 1,5 \\
\hline H3O & 1,0 & 0,5 & 0,5 & 2,0 \\
\hline H31 & 0,5 & 0,5 & 0,5 & 1,5 \\
\hline H32 & 0,5 & 1,0 & 1,0 & 2,5 \\
\hline H33 & 0,5 & 0,5 & 1,0 & 2,0 \\
\hline H34 & 1,0 & 0,5 & 0,5 & 2,0 \\
\hline H35 & 1,0 & 0,5 & 0,5 & 2,0 \\
\hline H36 & 1,0 & 1,0 & 1,0 & 3,0 \\
\hline
\end{tabular}

Nota: $\mathrm{H} 1$ a H36: hospitais identificados. Estrutura, Processo e Resultado: 21 pontos foram divididos entre os eixos, resultando em 7 pontos para cada eixo. Se pontuou 1 ou $2=0,0$; de 3 a $5=0,5 ; 6$ ou $7=1,0$ para o eixo correspondente. Nível de sedimentação: soma de todos os eixos. Se 0,0 ou $0,5=$ sedimentação incipiente; 1,0 ou 1,5= parcialmente Sedimentado; 2, 2,5 ou 3 = sedimentado.

Fonte: elaboração própria. 
A Itália apresentou três estudos 14,15,42 que corresponderam ao contexto de dois HGPE universitários sedimentados (H27 14,15 e H28 42) com unidades de ATS hospitalar, e H27 era parcialmente sedimentado 14 em 2005 e, em 2016, alcançou as dimensões de análise nos eixos estrutura, processo e resultado, que o caracterizaram um contexto sedimentado de ATS 15.

Em relação ao Brasil, dois estudos reportaram experiências de três hospitais com financiamento público (H8, H9 e H10 5,26), sendo um HGP e um HGPE de ensino e pesquisa com unidades de ATS hospitalar e um HGP universitário com um grupo matricial de ATS ou mini-ATS.

Dos seis hospitais com mais de um modelo organizacional de ATS, 83,3\% (5/6) apresentaram o nível sedimentado (H13 31, H20 27, H22 39, H26 41 e H30 43,44).

\section{Hospitais parcialmente sedimentados}

Nove hospitais de seis países apresentaram o nível parcialmente sedimentado (Tabela 1). Em relação ao eixo estrutura, nos hospitais H4, H5, H6, H7 e H23 havia grupos matriciais de ATS sem financiamento fixo para produção de estudos ou para elaboração de revisões rápidas chamadas de mini-ATS 25,47. Somente H23 destacou a presença de um formulário padronizado com critérios formais para planejamento, determinação das prioridades e atenção à tradução do conhecimento de forma aplicada às demandas do tomador de decisão 47. Todos os hospitais parcialmente sedimentados (H1 48, H4, H5, H6, H7 25, H23 47, H25 49, H29 50 e H31 51) destacaram ter equipes e partes interessadas incompletas e/ou com a presença de médicos em sua maioria. Em relação ao eixo processo, não foram informadas discussões aprofundadas sobre adaptabilidade do método utilizado ao contexto interno nem sobre replicabilidade externa $25,47,51$. Em relação ao eixo resultado, os hospitais parcialmente sedimentados apresentaram resultados parciais sobre o processo de incorporação de tecnologias em saúde 25,47,48,49,50,51.

Nos hospitais H1 48 e H25 49, havia unidades de ATS, e o H1 não especificou orçamento fixo, equipes capacitadas ou apoio administrativo para sua unidade 48 . Em relação ao eixo processo, ambos apresentaram processo formalizado com critérios para planejar e determinar prioridades adaptadas às configurações locais dos departamentos clínicos e da administração hospitalar 48,49. Somente H25 apresentou redução de custos e melhoria do processo, o que resultou na qualidade das tecnologias implementadas 49 .

No H29 havia uma unidade de ATS hospitalar que atuava com um comitê interno, no entanto não reportaram se a estrutura organizacional era institucionalizada ou se havia orçamento fixo para funcionamento da unidade de ATS 50. O H29 apresentou uma comissão multidisciplinar com grupos de trabalho colaborativos com outras instituições, para comparação das necessidades do território e avaliação de compra, aluguel ou descarte de tecnologias em saúde. Possui processo formal para ATS e produção de estudos para apoio processual. Reportaram que houve redução de custos e avaliação do preço médio de compra das tecnologias 50 .

No H31 havia comitês internos com financiamento, mas sem processos integrados para apoiar a governança de inclusão de novos serviços e tecnologias 51 . Destacaram a presença de equipes multidisciplinares capacitadas para realizar treinamentos, revisões rápidas e apoiar outros comitês e departamentos hospitalares. Reportaram que buscavam fazer alocação eficiente de recursos do orçamento hospitalar e realizar análise de evidências para formulação de políticas públicas sobre as tecnologias do hospital 51 .

Todos os hospitais de nível parcialmente sedimentado identificados da África do Sul 48, Austrália 25, Finlândia 47, Países Baixos 49, Singapura 51 e Itália 50 apresentaram perspectivas para sustentabilidade da ATS. Na África do Sul foram previstos esforços coordenados em nível nacional, regional e local 48. Nos hospitais australianos, as autoridades, organizações e empresas que supervisionam o setor privado reportaram promover fortemente a introdução da mini-ATS nos hospitais públicos e privados 25. Na Finlândia, informaram existir estratégias nacionais e locais sobre a efetividade na tomada de decisão do hospital, mas necessitam de maiores ações na prática hospitalar 47. Na Países Baixos, existe uma grande demanda para ATS hospitalar na produção de informações importantes para apoiar departamentos clínicos e conselhos do hospital ${ }^{49}$. No hospital de Singapura, a ATS está em fase inicial, entretanto os profissionais estão envolvidos com a comunidade científica nacional e internacional de ATS 51. Na Itália existe uma organização das comissões do hospital, mas ela ainda não foi institucionalizada 50 . 


\section{Características da atuação dos hospitais}

Todos os hospitais elaboraram critérios específicos que resultaram em ferramentas, sedimentadas e parcialmente sedimentadas, derivadas de relatórios, recomendações e/ou estudos de ATS, considerando a viabilidade, o conhecimento local e os critérios estabelecidos pela unidade. Os hospitais $\mathrm{H} 4$, H5, H6, H7 25 e H27 em sua fase inicial 14, possuíam critérios específicos, mas com processo ainda em desenvolvimento e sem padronização.

O principal método utilizado pela maioria dos hospitais foi o relatório de ATS. Essa ferramenta pode ser baseada em aspectos técnicos ou fundamentada em evidências de revisões da literatura científica para avaliação da relevância e aplicabilidade local 32. São emitidas recomendações com subsídios simples, transparentes e acessíveis 5,9,42, para sustentação da prática clínica e tomada de decisão em hospitais. A versão curta da ferramenta, chamada mini-ATS, possui uma breve avaliação das vantagens e desvantagens segundo um relato local 32 . Os relatórios podem ser submetidos a reuniões nacionais e internacionais, bem como a revistas científicas, e os resumos publicados no formato de boletins informativos $24,36,40$.

De forma geral, os processos de ATS precisam ter papel consultivo ${ }^{29}$, a fim de oferecer recomendações à gestão hospitalar no processo de aquisição ou retirada de tecnologias em saúde. Um conhecimento mais específico e adaptado ao contexto local pode também não apresentar replicabilidade do método a outras instituições, como destacado pelo hospital canadense Centro Hospitalar da Universidade de Sherbrooke 32,33,34,35. Nesse hospital, a utilização da ATS criou uma coerência organizativa específica ao processo local, que resultou em parcerias entre gestores, profissionais e representantes de usuários para produção de recomendações e desenvolvimento de políticas, mas que não é replicável a outros contextos por demandar recursos e habilidades específicas para condução dos estudos 35 .

\section{Barreiras para sedimentação da ATS hospitalar}

Trinta e dois hospitais, mesmo aqueles classificados como sedimentados, reportaram como principal barreira a capacidade para elaborar e manter o cumprimento das diretrizes e das formalidades locais para implementação da ATS hospitalar 5,9,11,12,13,14,15,16,24,25,26,27,28,29,30,31,32,33,34,35,36,37,38,39,41,42,43,44,45, 46,47,50,51. Somente H1, H10, H24 e H25 não reportaram capacidade instalada como barreira 26,40,48,49.

Outros aspectos que limitavam e/ou dificultavam a sedimentação da ATS hospitalar foram reportados pelos hospitais, como a falta de transparência, planejamento e agilidade no processo de aquisição de tecnologias (27/36); inércia profissional e resistência à mudança (26/36); não adesão às ferramentas ATS ou outras derivações de apoio à decisão local (21/36); capacitações, orientações e habilidades técnicas insuficientes (21/36); falta de reconhecimento interno e externo (20/36); falta de apoio administrativo, financeiro ou governamental (16/36); garantia da decisão baseada em evidências, sem influências (13/36); gerência desacreditada sobre as decisões tomadas coletivamente (13/36); escassez de evidências qualificadas (11/36); e, por fim, a falta de dedicação exclusiva, relacionada ao tempo e rotatividade de profissionais $(8 / 36)$

Os modelos organizativos presentes nos hospitais precisam de recursos constantes para que a prática da ATS hospitalar contribua para qualidade e segurança na adoção das tecnologias. O abandono de práticas inovadoras pelo não financiamento, reconhecimento e/ou acompanhamento dos processos foi citado em um estudo 31 .

Segundo os estudos selecionados, os gestores que não possuem financiamento interno podem optar, conforme o seu contexto, por outras fontes de financiamento, como reembolso de procedimentos 26; busca por apoio financeiro e orçamentos hospitalares 15; financiamento externo 32; subsídios competitivos 27,37; buscas de recursos em departamentos de saúde ou conselhos regionais 16,44 e financiamentos de organizações nacionais para área de ATS 29,49. 


\section{Discussão}

Esta revisão de escopo identificou níveis de sedimentação em 36 hospitais, sendo 75\% (27/36) classificada como sedimentada porque completou critérios de estruturação de modelos organizativos de ATS, apresentou etapas do processo de ATS e demonstrou a implementação dos resultados práticos para o nível hospitalar.

Resultados práticos para gestão hospitalar estavam relacionados com a própria capacidade da equipe hospitalar mostrar valor ao campo da ATS no âmbito da gestão hospitalar e quando tais equipes também contribuíam com as políticas gerais de cobertura adotadas em seus países. Hailey et al. $52 \mathrm{e}$ Favaretti et al. 53 corroboram esses achados ao apresentarem estudos sobre impactos da ATS em nível local e nacional. Novaes et al. 54 reportam que a adoção de processos deliberativos, quando inseridos em estruturas de avaliação de valor, pode imprimir mais legitimidade, tanto nas deliberações como na priorização de tecnologias.

A alocação de recursos fixos para os modelos organizativos presentes nos hospitais foi especificada em 17 hospitais sedimentados. Esse achado pode ser corroborado por Attieh et al. 55, que em revisão sistemática reportou efeitos e repercussões da adoção de ATS por hospitais, mostrando que, ao trazer economias para os hospitais, a ATS proporcionou a importância da continuidade do trabalho por profissionais dedicados e sustentação de modelos organizativos.

A sustentabilidade do apoio e do compromisso gerencial em manter as estruturas de ATS hospitalar foram fatores-chave para crescimento interno e externo segundo alguns estudos 9,24 , que reportaram experiências de ATS hospitalar. Esses fatores também foram confirmados por Francisco \& Malik 7 , ao analisarem a experiência dos núcleos de ATS no Brasil, reportando que a sustentação da ATS hospitalar ao longo do tempo está relacionada ao reforço da gestão estratégica do hospital.

No que tange à experiência de outros núcleos de ATS no Brasil, para sustentabilidade são necessários investimentos contínuos para aumento da produção, educação continuada e adesão, legitimando as ações gestoras, e que estas sejam direcionadas a políticas de ATS relevantes ao contexto experienciado 5,6 .

O processo de elaboração dos critérios para solicitação de relatórios de ATS e a emissão de recomendações esteve presente em 14 dos hospitais identificados. Entre os problemas apresentados, estavam a ausência de formalização, cuja consequência foi abrir caminho para conflitos de interesses e desconfianças 47 , e influências externas na emissão de recomendações 28 . Grenon et al. 46 reportaram que esses problemas ocorrem quando não há procedimentos padronizados para desenvolvimento dos relatórios de ATS e que tais processos precisam ser desenvolvidos no contexto local e de maneira formalizada.

\section{Limitações do estudo}

Essa revisão de escopo trouxe experiências identificadas por meio de publicações em periódicos nacionais e internacionais, não tendo sido feitas visitas técnicas ou entrevistas com informantes-chave dos hospitais analisados, consistindo em um estudo exploratório. Embora tenha se desenvolvido estratégia de busca sensibilizada, busca manual e buscas em anais de conferências especializadas na área, muitos resumos publicados nos anais desses eventos científicos não puderam ser utilizados por não apresentarem informações completas dos hospitais a que se referiam. Somente foram selecionados nesta revisão de escopo estudos que abordavam a ATS especificamente em um contexto hospitalar e excluídos estudos que tratavam do tema em âmbito nacional ou de forma geral, sem citar as experiências singulares de cada hospital. O processo de extração de dados dos artigos, embora realizado por um autor, foi revisado em reuniões com os demais autores para dirimir dúvidas de classificação em relação às dimensões de sedimentação. Alguns hospitais podem ter sido prejudicados na avaliação devido à falta de informações específicas sobre a estrutura, processo e resultados da ATS nos hospitais identificados, embora os autores tenham buscado informações em websites. 


\section{Implicações para a prática}

O estudo contribuiu para estruturação de ações de ATS em hospitais do Sistema Único de Saúde (SUS). Conhecer as experiências e o nível de sedimentação da ATS hospitalar, em contexto nacional e internacional, contribui para maior compreensão do campo, uma vez que os estudos da revisão de escopo apontam sustentabilidade, crescimento e evolução da ATS hospitalar em países com e sem tradição no tema. Assim, o estudo apresentou dimensões que servirão de base para mapeamento de capacidades instaladas de hospitais e novos estudos sobre ATS em hospitais.

\section{Conclusões}

Esta revisão de escopo mostrou dimensões e níveis de sedimentação em 36 hospitais que adotaram ATS nos seus processos gestores, identificados com a busca extensiva de experiências nacionais e internacionais. A maioria foi classificado como sedimentado porque completou critérios de estruturação de modelos organizativos de ATS, apresentou etapas do processo da ATS e demonstrou os resultados práticos para o nível hospitalar. Observou-se também que em todo o processo de sedimentação da ATS hospitalar existem desafios presentes, desde a implementação de novas práticas clínicas e gerenciais até a sustentação das estruturas e processos ao longo do tempo.

Em hospitais com a ATS parcialmente sedimentada, as barreiras iniciais relativas aos aspectos estruturais estavam relacionadas aos recursos financeiros, físicos e humanos disponíveis e ao processo de adesão da ferramenta aplicada ao contexto da gestão estratégica. Nos hospitais com ATS sedimentada, a principal barreira foi imprimir impacto aos resultados, explicados pela própria escassez de evidências para tópicos avaliados, pela falta de capacitações contínua e pelo descumprimento de diretrizes estabelecidas no processo de avaliação e decisão, influenciando o reconhecimento interno e externo dos resultados obtidos com a ATS. Espera-se que as dimensões analisadas e os achados sirvam de base para criar estratégias de implementação da ATS em hospitais.

\section{Colaboradores}

J. P. S. Galdino, E. B. Camargo e F. T. S. Elias contribuíram na concepção e projeto, análise e interpretação dos dados, na redação do artigo, revisão crítica relevante do conteúdo intelectual e na aprovação final da versão a ser publicada. Os autores são responsáveis por todos os aspectos do trabalho na garantia da exatidão e integridade de qualquer parte da obra.

\section{Conflito de interesses}

Os autores declaram não ter conflito de qualquer natureza em relação à condição do estudo.

\section{Informações adicionais}

ORCID: Johnathan Portela da Silva Galdino (00000002-3994-1479); Erika Barbosa Camargo (00000003-1482-8282); Flavia Tavares Silva Elias (00000002-7142-6266).

\section{Agradecimentos}

Agradecemos às especialistas que participaram do grupo focal para criação da pontuação de classificação de nível de sedimentação e apoio nas buscas da literatura. O estudo contou com apoio do projeto de cooperação da Fundação Oswaldo Cruz (Fiocruz) com a Agência Nacional de Vigilância Sanitária (Anvisa): Ações para Apoio à Governança Regulatória de Produtos Sujeitos à Vigilância Sanitária. Fiocruz Brasília. 


\section{Referências}

1. Sampietro-Colom L, Martin J. Hospital-based health technology assessment: the next frontier. In: Sampietro-Colom L, Martin J, editors. Hospital-based health technology assessment: the next frontier for health technology assessment. Cham: Springer; 2017. p. 3-11.

2. Sampietro-Colom LLK, Cicchetti AKK, Pasternack IFB, Rosenmöller MWC, Kahveci RWJ, Kiivet RA et al. The AdHopHTA handbook 2015. http://www.adhophta.eu/sites/ files/adhophta/media/adhophta_handbook_ website.pdf (acessado em 20/Dez/2020).

3. Sampietro-Colom L, Lach K, Pasternack I, Wasserfallen J-B, Cicchetti A, Marchetti M, et al. Guiding principles for good practices in hospital-based health technology assessment units. Int J Technol Assess Health Care 2016; 31:457-65.

4. Souza NP, Lira PIC, Fontbonne A, Pinto FC de L, Cesse EAP. (Mal)nutrition and the new epidemiological trend in a context of development and inequalities. Ciênc Saúde Colet 2017; 22:2257-66.

5. Nunes AA, Mello LM, Ana LW, Marques PMA, Dallora MEL, Martinez EZ, et al. Avaliação e incorporação de tecnologias em saúde: processo e metodologia adotados por um hospital universitário de alta complexidade assistencial. Cad Saúde Pública 2013; 29 Suppl 1:S179-86.

6. Aguiar Pereira CC, Dos Santos Rabello R, Elias FTS. Hospital-based health technology assessment in Brazil: an overview of the initial experiences. Int J Technol Assess Health Care 2017; 33:227-31

7. Francisco F, Malik AM. Aplicação de Avaliação de Tecnologias em Saúde (ATS) na tomada de decisão em hospitais. J Bras Econ Saúde 2019; 11:10-7.

8. Banta D, Jonsson E. History of HTA: introduction. Int J Technol Assess Health Care 2009; 25 Suppl 1:1-6.

9. Demirdjian G. A 10-year hospital-based health technology assessment program in a public hospital in Argentina. Int J Technol Assess Health Care 2015; 31:103-10.

10. Francisco FR, Malik AM. Aplicação de avaliação de tecnologias em saúde (ATS) na tomada de decisão em hospitais. J Bras Econ Saúde 2019; 11:10-7.

11. McGregor M, Brophy JM. End-user involvement in health technology assessment (HTA) development: a way to increase impact. Int J Technol Assess Health Care 2005; 21:263-7.

12. McGregor M. The Health Technology Assessment Unit (TAU) of the McGill University Health Centre (MUHC) (Canada). In: Sampietro-Colom L, Martin J, editors. Hospital-based health technology assessment: the next frontier for health technology assessment. Cham: Springer; 2017. p. 167-71.
13. Almeida ND, Mines L, Nicolau I, Sinclair A, Forero DF, Brophy JM, et al. A framework for aiding the translation of scientific evidence into policy: the experience of a hospital-based technology assessment unit. Int J Technol Assess Health Care 2019; 35:204-11.

14. Catananti C, Cicchetti A, Marchetti M. Hospital-based health technology assessment: the experience of Agostino Gemelli University Hospital's HTA Unit. Ital J Public Health 2005; 2:23-8.

15. Marchetti M, Cicchetti A. The HTA and innovation unit at the A. Gemelli University Hospital (Italy). In: Sampietro-Colom L, Martin J, editors. Hospital-based health technology assessment: the next frontier for health technology assessment. Cham: Springer; 2017. p. 85-94.

16. Kosherbayeva L, Hailey D, Kurakbaev K, Tsoy A, Zhuzzhanov O, Donbay A, et al. Implementation of health technology assessment work in a hospital in Kazakhstan. Int J Technol Assess Health Care 2016; 32:78-80.

17. Donabedian A. Criteria and standards for quality assessment and monitoring. Qual Rev Bull 1986; 12:99-108.

18. Donabedian A. The seven pillars of quality. Arch Pathol Lab Med 1990; 114:1115-8.

19. Nascimento GC, Rodrigues VJ, Megliorini E. Conceitos da teoria institucional: fonte propulsora de evolução para a gestão de desempenho. In: XVII Congresso Brasileiro de Custos. https://anaiscbc.emnuvens.com.br/anais/ article/viewFile/761/761 (acessado em 20/ Dez/2020).

20. Peters MDJ, Godfrey C, McInerney P, Munn Z, Tricco AC, Khalil, H. Scoping reviews. In: Aromataris E, Munn Z, editors. Joanna Briggs Institute reviewer's manual. https://wiki.jbi.global/display/MANUAL/ Chapter+11\%3A+Scoping+reviews (acessado em 20/Dez/2020).

21. Tricco AC, Lillie E, Zarin W, O’Brien KK, Colquhoun H, Levac D, et al. PRISMA Extension for Scoping Reviews (PRISMA-ScR): checklist and explanation. Ann Intern Med 2018; 169:467-73.

22. Braga Neto FC, Barbosa PR, Santos IS, Oliveira CMF. Atenção hospitalar: evolução histórica e tendências. In: Giovanella L, Escorel S, Lobato LVC, Noronha JC, Carvalho AI, organizadores. Políticas e sistema de saúde no Brasil. Rio de Janeiro: Editora Fiocruz/Centro Brasileiro de Estudos de Saúde; 2008. p. 577-608.

23. Samprietro-Colom L, Lach K, Escolar I, Sroka S, Soto M, Cicchetti A, et al. A handbook and a toolkit for hospital-based health technology assessment. Value Health 2015; 18:A556. 
24. Demirdjian G, Kurtzbart R, Hernandez R. Hospital-based HTA in Argentina: the Hospital Garrahan and Hospital El Cruce experiences. In: Sampietro-Colom L, Martin J, editors. Hospital-based health technology assessment: the next frontier for health technology assessment. Cham: Springer; 2017. p. 247-61.

25. Saaid HB, Stewart D, England I, Parmar N. The impact of health technology assessment on decision-making orocesses in public versus not-for-profit private hospitals. Am Med J 2011; 2:72-8.

26. Santos M, Magliano C, Kelles DB, Kelles SMB, Stein A. The role of hospitals in HTA in Brazil. In: Sampietro-Colom L, Martin J, editors. Hospital-based health technology assessment: the next frontier for health technology assessment. Cham: Springer; 2017. p. 239-46.

27. Sampietro-Colom L, Soto M, García C, Benot $\mathrm{S}$. Hospital-based HTA in three Spanish hospitals. In: Sampietro-Colom L, Martin J, editors. Hospital-based health technology assessment: the next frontier for health technology assessment. Cham: Springer; 2017. p. 57-69.

28. Wasserfallen J-B, Pinget C. Hospital-based HTA in Switzerland. In: Sampietro-Colom L, Martin J, editors. Hospital-based health technology assessment: the next frontier for health technology assessment. Cham: Springer; 2017. p. 77-83.

29. Kidholm K, Ølholm AM. Hospital-based HTA in Denmark. In: Sampietro-Colom L, Martin J, editors. Hospital-based health technology assessment: the next frontier for health technology assessment. Cham: Springer; 2017. p. 39-44.

30. Kahveci R, Tütüncü T, Yüksek YN, Küçük EÖ, Koç EM, Zengin N. Hospital-based HTA in Turkey. In: Sampietro-Colom L, Martin J, editors. Hospital-based health technology assessment: the next frontier for health technology assessment. Cham: Springer; 2017. p. 95-105.

31. Lepanto L. Hospital-based HTA at the Centre Hospitalier de l'Université de Montréal (Canada). In: Sampietro-Colom L, Martin J, editors. Hospital-based health technology assessment: the next frontier for health technology assessment. Cham: Springer; 2017. p. 173-83.

32. Bellemare CA, Fisette J-F, Poder TG, Bédard SK, Dagenais P. The health technology assessment unit of the Centre hospitalier universitaire de Sherbrooke (Canada). In: SampietroColom L, Martin J, editors. Hospital-based health technology assessment: the next frontier for health technology assessment. Cham: Springer; 2017. p. 185-200.

33. Poder TG. Using the health technology assessment toolbox to facilitate procurement: the case of smart pumps in a Canadian hospital. Int J Technol Assess Health Care 2017; 33:54-62.
34. Poder TG, Bellemare CA, Bédard SK, Fisette J-F, Dagenais P. Impact of health technology assessment reports on hospital decision makers - 10-year insight from a hospital unit in Sherbrooke, Canada: impact of health technology assessment on hospital decisions. Int J Technol Assess Health Care 2018; 34:393-9.

35. Poder TG, Beffarat M, Benkhalti M, Ladouceur G, Dagenais P. A discrete choice experiment on preferences of patients with low back pain about non-surgical treatments: identification, refinement and selection of attributes and levels. Patient Prefer Adherence 2019; 13:933-40.

36. Rhainds M, Asselin G, Coulombe M. CHU de Québec - Université Laval: 10-years' experience in hospital-based HTA (Canada). In: Sampietro-Colom L, Martin J, editors. Hospital-based health technology assessment: the next frontier for health technology assessment. Cham: Springer; 2017. p. 201-11.

37. Ungar WJ. Technology Assessment at SickKids (TASK): a health technology assessment research unit devoted to child health in Canada. In: Sampietro-Colom L, Martin J, editors. Hospital-based health technology assessment: the next frontier for health technology assessment. Cham: Springer; 2017. p. 153-65.

38. Avdeyev A, Tabarov A, Akhetov A, Shanazarov N, Hailey D, Kaptagayeva A, et al. Hospitalbased health technology assessment in $\mathrm{Ka}$ zakhstan: 3 years' experience of one unit. Int J Technol Assess Health Care 2019; 35:436-40.

39. Mitchell MD, Williams K, Brennan PJ, Umscheid CA. Integrating local data into hospitalbased healthcare technology assessment: two case studies. Int J Technol Assess Health Care 2010; 26:294-300.

40. Barna A, Fahlgren B, Charpentier E, TaronBrocard C, Guillevin L. The "Comité d'Evaluation et de Diffusion des Innovations Technologiques" (CEDIT) in France. In: Sampietro-Colom L, Martin J, editors. Hospital-based health technology assessment: the next frontier for health technology assessment. Cham: Springer; 2017. p. 71-6.

41. Tal O, Booch M, Bar-Yehuda S. Hospital staff perspectives towards health technology assessment: data from a multidisciplinary survey. Health Res Policy Syst 2019; 17:72.

42. Miniati R, Frosini F, Cecconi G, Dori F, Gentili GB. Development of sustainable models for technology evaluation in hospital. Technol Health Care 2014; 22:729-39.

43. Munn SR. Hospital-based health technology assessment: insights from New Zealand. Pharmacoeconomics 2014; 32:815-7. 
44. Fitzgerald A, Streat S, McAleese C, Munn S. Hospital-based HTA in New Zealand. In: Sampietro-Colom L, Martin J, editors. Hospital-based health technology assessment: the next frontier for health technology assessment. Cham: Springer; 2017. p. 305-12.

45. Jivegård L, Bergh C, Kindblom J, Samuelsson O, Sjögren P, Sjövall H, et al. Activity-based HTA: hospital-based HTA performed by clinicians with support and quality control, the Sahlgrenska University Hospital HTA-Centrum Experience (Sweden). In: Sampietro-Colom L, Martin J, editors. Hospital-based health technology assessment: the next frontier for health technology assessment. Cham: Springer; 2017. p. 15-28.

46. Grenon X, Pinget C, Wasserfallen J-B. Hospital-based health technology assessment (HBHTA): a 10-year survey at one unit. Int J Technol Assess Health Care 2016; 32:116-21.

47. Pasternack RP, Iris R. HTA activities in finnish hospitals. In: Sampietro-Colom L, Martin J, editors. Hospital-based health technology assessment: the next frontier for health technology assessment. Cham: Springer; 2017. p. 29-37.

48. Mueller DB, Govender M. HTA in a public hospital in South Africa. In: Sampietro-Colom L, Martin J, editors. Hospital-based health technology assessment: the next frontier for health technology assessment. Cham: Springer; 2017. p. 263-72.

49. van der Wilt GJ, Rovers M, Oortwijn W, Grutters J. Hospital-based HTA at Radboud University Medical Centre in the Netherlands: welcome to reality. In: Sampietro-Colom L, Martin J, editors. Hospital-based health technology assessment: the next frontier for health technology assessment. Cham: Springer; 2017. p. 45-55.
50. Manzi P, Barberini P, Dori F, Cecchini A. Hospital based health technology assessment: an example from Siena. Farmeconomia. Health Economics and Therapeutic Pathways 2015; 16:7-13.

51. Pwee KH, Chow WL. Hospital-based HTA in a public-sector tertiary hospital in Singapore. In: Sampietro-Colom L, Martin J, editors. Hospital-based health technology assessment: the next frontier for health technology assessment. Cham: Springer; 2017. p. 273-82.

52. Hailey D, Werkö S, Rosén M, MacPherson K, Myles S, Gallegos Rivero V, et al. Influence of health technology assessment and its measurement. Int J Technol Assess Health Care 2016; 32:376-84.

53. Favaretti C, Cicchetti A, Guarrera G, Marchetti M, Ricciardi W. Health technology assessment in Italy. Int J Technol Assess Health Care 2009; 25 Suppl 1:127-33.

54. Novaes HMD, De Soárez PC. A Avaliação das Tecnologias em Saúde: origem, desenvolvimento e desafios atuais. Panorama internacional e Brasil. Cad Saúde Pública 2020; 36:e00006820.

55. Attieh R, Gagnon M-P. Implementation of lo$\mathrm{cal} /$ hospital-based health technology assessment initiatives in low- and middle-income countries. Int $\mathrm{J}$ Technol Assess Health Care 2012; 28:445-51. 


\section{Abstract}

The aim of this study was to analyze the level of sedimentation of hospital-based health technology assessment (HTA) in diverse contexts. A scoping review was conducted according to the methodology of the Joanna Briggs Institute, whose data analysis model consisted of the combination of Donabedian's structure, process, and outcome categories and the dimensions of the project Adopting Hospital Based Health Technology Assessment in European Union (AdHopHTA). We identified 270 studies, and after removing duplicates and reading full texts, 36 references met the eligibility criteria. Thirty-six hospitals were identified, of which there were 24 large-scale hospitals with extra bed capacity. Twenty-three hospitals were affiliated with universities. Canada stood out with five university hospitals, four of which with public funding. Half of the identified hospitals had hospital-based HTA units $(18 / 36)$. Hospitals with sedimented levels of HTA corresponded to $75 \%$ of the sample (27/36), and the remainder had partially sedimented HTA, or 25\% of the hospitals in the review (9/36). There were no hospitals with incipient sedimentation. Measuring the level of HTA sedimentation in the hospitals contributed to understanding how their participation has occurred in the field of hospitalbased HTA. This study revealed the importance of identifying factors such as sustainability, growth, and evolution of hospital-based HTA in countries with and without a tradition in this field.

Biomedical Technology Assessment; Hospitals; Health Management; Decision Making;

Governance

\section{Resumen}

El objetivo del estudio fue analizar el nivel de sedimentación en la evaluación de tecnologías de salud (ATS) en hospitales dentro de diversos contextos. Se realizó una revisión de alcance, según la metodología del Instituto Joanna Briggs, cuyo modelo de análisis de datos estuvo compuesto por la combinación de las dimensiones: estructura, proceso y resultado de Donabedian, así como las dimensiones del proyecto Adopting Hospital Based Health Technology Assessment in European Union (AdHopHTA). Se identificaron 270 estudios, tras la eliminación de duplicados y lectura de textos completos, 36 referencias atendieron a los criterios de elegibilidad. Se identificaron treinta $y$ seis hospitales, 24 de los cuales, de gran porte, con capacidad extra de camas. Veintitrés hospitales contaban con vínculos universitarios. Canadá merece una mención especial, con cinco hospitales universitarios, cuatro de los cuales con financiación pública. La mitad de los hospitales identificados tenían unidades de ATS hospitalaria (18/36). Hospitales con un nivel sedimentado correspondieron a un $75 \%$ (27/36), y parcialmente sedimentado a un $25 \%(9 / 36)$. No hubo hospital con sedimentación incipiente. Medir el nivel de sedimentación de la ATS en los hospitales identificados contribuye al entendimiento de cómo se produce la inserción en el campo de la ATS hospitalaria. En este estudio, se mostró la importancia de identificar factores como: sostenibilidad, crecimiento y evolución de la ATS hospitalaria en países con y sin tradición en este ámbito.

Evaluación de la Tecnología Biomédica; Hospitales; Gestión en Salud; Toma de Decisiones; Gobernanza
Recebido em 25/Dez/2020

Versão final reapresentada em 18/Mai/2021 Aprovado em 28/Mai/2021 